\title{
Manufatura Digital no Planejamento da Automação da Usinagem de COMPONENTES POWERTRAIN
}

\author{
Diego Barbosa Pratis Santos, Eduardo Eustáquio Ferreira Barbosa \\ FIAT Chrysler Automobiles
}

E-mails: diego.pratis@fcagroup.com, eduardo.barbosa@fcagroup.com

\section{RESUMO}

Recursos tecnológicos estão cada vez mais presentes em processos produtivos manufaturados, metal mecânico, medicina, alimentícia, etc. Manufatura digital aplicada no desenvolvimento de projetos de processos industriais vem gerando melhorias substanciais na eficiência dos processos produtivos. Empresas destes setores têm investido em recursos humanos, treinamento, hardware e software para mitigar problemas relacionados à produtividade através da representação virtual do ambiente fabril em 3D dinâmico dando suporte à criação, reutilização e validação dos processos. Dado o cenário de expressiva competitividade do mercado e da inexistência de fronteiras geográficas para a comercialização dos produtos, as organizações se mantem competitivas por meio da redução dos custos de transformação inerentes à manufatura. A modelagem, animação e simulação dos processos otimiza o seu custo do ciclo de vida e antecipa os problemas na construção dos equipamentos na fase de projeto, minimizando ou anulando os custos relativos a modificações e retrabalhos durante a instalação e comissionamento. O presente estudo propõe-se avaliar a aplicação da manufatura digital na automação de uma linha de usinagem de peças powertrain (bloco e cabeçote motor), e ainda os impactos no desenvolvimento de um projeto.

\section{INTRODUÇÃO}

A conceituação de Sistemas Ciber-Físicos (CPS) foi pela primeira vez definido pelo Dr. James Truchard, CEO da National Instruments em 2006, baseado na representação virtual de um processo de manufatura em softwares. Posteriormente em 2012 foi formado na Alemanha um grupo especializado para discutir os futuros chamada Industria 4.0, mais conhecida como a quarta revolução industrial. No Brasil alguns centros de pesquisas, universidades e empresas já iniciaram trabalhos direcionados ao novo paradigma industrial. Em junho de 2014 o governo criou o Programa Nacional de Plataformas do Conhecimento, sinalizando investimentos direcionados à manufatura avançada, integrante da Industria 4.0, e uma das dez área de tecnologia prioritárias para o futuro.

A manufatura digital [1], liderada principalmente pela indústria automotiva, é parte de um novo paradigma industrial, onde os produtos estão cada vez direcionados ao mercado consumidor. Percebe-se atualmente que a modularidade e a variedade de itens opcionais faz com que seja aumentada também a complexidade dos processos de manufatura, que por outro lado deve atender aos desejos do mercado em um tempo cada vez mais curto [2].

É neste cenário que a manufatura digital está inserida, como um recurso tecnológico para o planejamento de um processo, verificação da produtividade e também verificação da 
eficiência dos sistemas de manufatura. O resultado visa a produção de produtos em tempo e custos otimizados, por meio da virtualização do seu processo de fabricação anteriormente a criação real dos mesmos.

Para Chang o "planejamento do processo produtivo é uma função vital nas indústrias de manufatura, pois fornece as informações necessárias para a transformação de matéria-prima em produtos finais" [3].

As máquinas dos processos de manufatura apresentam, sob a ótica de produtividade, características semelhantes relacionadas à eficiência técnica. Cada célula de manufatura possui variáveis que impactam diretamente na redução da eficiência técnica do equipamento e, consequentemente, na produtividade global da fábrica. Geralmente, as linhas de usinagem dos componentes "powertrain" bloco e cabeçote motor, nos seus processos, apresentam tipologias de máquinas como centros de usinagem $\mathrm{CNC}$, lavadoras de alta pressão, testes de estanqueidade e células de montagem. Cada uma destas células possuem Meantime Between Failure (MTBF), Meantime to Repair (MTTR) [4], frequentes atividades de limpeza e inspeção, set-up e, no caso de centros de usinagem $\mathrm{CNC}$, trocas de ferramentas. Estes quando combinados de forma mútua no dia-a-dia do processo produtivo somado com os padrões estocásticos destes eventos, podem gerar resultados não satisfatórios quando comparados ao escopo original do projeto.

O desenvolvimento de um planejamento adequado do processo de automação através da manufatura digital tem propiciado cenários assertivos na comparação dos resultados obtidos do ambiente virtual com o escopo e, após a validação, projeto e construção, com o real. Quando se leva em consideração a disposição dos equipamentos baseado no fluxo do processo (configuração paralela ou em série, por exemplo) o Overall Equipment Effectiveness (O.E.E) da linha [5] pode ser reduzido, pois todos os eventos de impacto na eficiência técnica serão combinados de forma conjunta com o meio de automação da movimentação das peças entre as estações. Os resultados extraídos do planejamento do processo de automação também definirá as futuras especificações dos sistemas robóticos e as programações inseridas.

O modelamento e simulação virtual de um novo processo conta com a disposição dos dados teóricos declarados pelos fabricantes normalmente fornecidos antes do término da negociação comercial, que muita das vezes são subestimados e não refletem a contribuição da gestão da manutenção da planta na eficiência técnica global. O planejamento da automação requer um estudo de diversos cenários virtuais para a comparação dos dados teóricos, obtidos através dos fornecedores, com os dados coletados de equipamentos em operação com o mesmo padrão ou similaridade processual.

Uma das ferramentas de simulação virtual aplicada à manufatura digital é a Discrete Event Simulation (DES), sendo esta a principal contribuição deste trabalho. Na simulação por eventos discretos os componentes de um sistema, dentre eles máquinas, operadores e materiais, são modelados como "objetos" e o estado destes variam de acordo com eventos específicos que ocorrem dentro de um espaço de tempo, como por exemplo a quebra de maquina, a troca de turno ou qualquer instante de downtime. Cada "objeto" é parametrizado com os atributos de disponibilidade, tempo de ciclo, dimensões de materiais, tempo para realização de alguma atividade ou confiabilidade [6]. A tabela 1 apresenta a composição analítica para uma modelagem utilizando DES. 
Tabela 1 - Composição de uma Simulação por Eventos Discretos

\begin{tabular}{c|c|c|c}
\hline Sistema & Objetos & Atributos & Eventos \\
\hline linha de produção & $\begin{array}{c}\text { máquinas, operadores, } \\
\text { materiais. }\end{array}$ & $\begin{array}{c}\text { dimensionamentos, } \\
\text { confiabilidade, tempos de } \\
\text { ciclo. }\end{array}$ & $\begin{array}{c}\text { quebra de máquina, } \\
\text { troca de turno, setup. }\end{array}$ \\
\hline
\end{tabular}

\section{INFORMAÇÕES GERAIS}

O modelamento computacional de uma linha de produção através da simulação a eventos discretos contribui significativamente para o projeto do processo de manufatura por permitir aos engenheiros um maior entendimento das particularidades do sistema. Contudo a relevância destas informações é constatada somente quando o estudo é realizado na fase de planejamento ou projeto básico, o que permite incorporar os resultados da simulação na especificação técnica dos equipamentos.

O presente estudo avaliou, através de procedimento experimental, se a produtividade das linhas de usinagem de bloco e cabeçote motor atenderiam a demanda da organização. Durante o experimento a lógica de automação entre as células de manufatura foi variada com o objetivo de maximizar a produtividade. Paralelamente a este, os resultados obtidos através dos atributos provenientes de eficiência técnica teórica foram comparados com dados reais de eficiência técnica (obtidos através de benchmarking).

Como as linhas de usinagem foram concebidas para produção de dois tipos distintos de bloco e cabeçote motor, a automação destes processos precisa garantir além da flexibilidade operativa nos volumes requeridos, a futura possibilidade de acréscimo de novas tipologias de produtos. Neste contexto foi definida a tecnologia de automação através de robô antropomorfo de 7 eixos, com o deslocamento através de trilhos sobre as máquinas. Este conceito apresenta algumas vantagens quando comparado com os sistemas tradicionais de automação como modularidade, eficiência e, principalmente, flexibilidade.

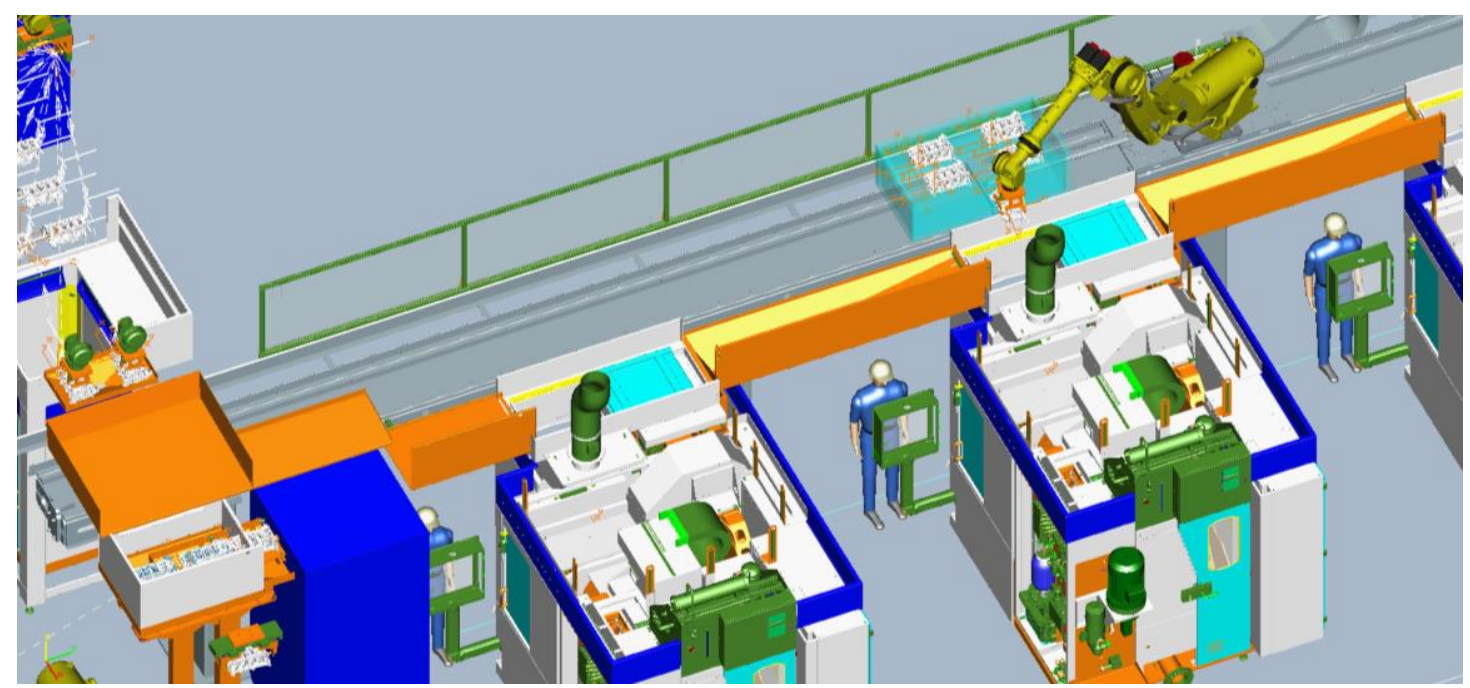

Figura 1: Robô antropomorfo de 7 eixos com deslocamento sobre trilhos (Fonte: autor). 


\section{PROCEDIMENTO EXPERIMENTAL}

Um software padrão para simulação de eventos discretos foi utilizado no desenvolvimento dos procedimentos experimentais descritos a seguir. O principal KPI do modelo simulado foi a produtividade horária do sistema, seguidos da eficiência global, margem de produtividade e lead time das peças na linha. Para cada um destes foram avaliados três cenários distintos: ideal, eficiência teórica e eficiência real. No cenário denominado "ideal" os atributos parametrizados no sistema foram os tempos ciclos dos centros de usinagem e máquinas auxiliares, para cada tipo, tempos de carga e descarga das peças, velocidade e aceleração nominal do robô antropomorfo e mix de produção. Não foram incluídos neste cenário as estatísticas de MTBF e MTTR, pois o objetivo com esta simulação foi compreender o comportamento do sistema e a eficiência da automação com robô sobre trilhos em um cenário sem falhas.

No cenário de "eficiência teórica" foram adicionados aos atributos descritos no parágrafo anterior a troca de ferramentas, setup, atividades de limpeza, MTBF e MTTR declarado pelos fabricantes das máquinas. A diferença deste para o cenário ideal é a introdução das variáveis aleatórias com padrão estocástico, que são as estatísticas de falhas. Apesar dos fabricantes de máquinas e equipamentos declararem estes dados baseado no know-how do seu equipamento, não tem como ter a precisão de quando estes eventos irão ocorrer.

Por último, o cenário "eficiência real" conta com todos os atributos elencados no cenário de eficiência teórica somados com percentual de refugo, além de micro paradas. Esta última estatística de falha é obtida através de coleta de dados nas plantas de manufatura decorrente de alarmes, inspeções, etc. O MTBF e MTTR parametrizados neste foram obtidos através de estudo de benchmarking realizados em diferentes plantas de manufatura observando equipamentos dos mesmos fabricantes e tipologia de processo. A eficiência técnica do equipamento sofre alteração em função da gestão de manutenção, que é particular em cada planta e organização. A diagnose da falha, disponibilidade de peças de reposição, procedimentos para intervenção e análise de risco alteram de forma significativa as estatísticas originais dos fabricantes. $\mathrm{O}$ quadro 1 resume os cenários descritos bem como os atributos para contidos em cada um destes.

Quadro 1 - Resumo dos cenários simulados e definição de atributos

\begin{tabular}{|c|c|c|c|}
\hline & \multicolumn{3}{|c|}{ Cenários } \\
\hline & Ideal & Eficiência técnica & Eficiência real \\
\hline 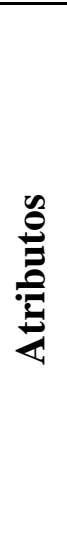 & $\begin{array}{c}\text { tempo ciclo } \\
\text { carga e descarga } \\
\text { velocidade/aceleração } \\
\text { mix produção }\end{array}$ & $\begin{array}{c}\text { tempo ciclo } \\
\text { carga e descarga } \\
\text { velocidade/aceleração } \\
\text { mix produção } \\
\text { troca ferramentas } \\
\text { setup } \\
\text { limpeza } \\
\text { MTBF } \\
\text { MTTR }\end{array}$ & $\begin{array}{c}\text { tempo ciclo } \\
\text { carga e descarga } \\
\text { velocidade/aceleração } \\
\text { mix produção } \\
\text { troca ferramentas } \\
\text { setup } \\
\text { limpeza } \\
\text { MTBF } \\
\text { MTTR } \\
\text { \% refugo } \\
\text { micro paradas }\end{array}$ \\
\hline
\end{tabular}


Em cada cenário foram simulados 265 dias de produção com 3 turnos diários de produção. Os eventos foram parametrizados no simulador de eventos discretos com distribuição de probabilidade constante (troca de ferramentas, setup, limpeza e \% de refugos) e exponencial negativa (MTBF e MTTR). Foi utilizado o algoritmo gerador de números aleatórios do próprio sistema para a ocorrência das falhas durante o intervalo de simulação.

\section{RESULTADOS}

Por meio dos atributos utilizados no simulador de eventos discretos, subdividindo as entradas em três cenários: "ideal", "eficiência técnica" e "eficiência real" foi possível traçar pontos de análises que descrevem o comportamento de cada KPI, conforme descritos no Gráfico 1.

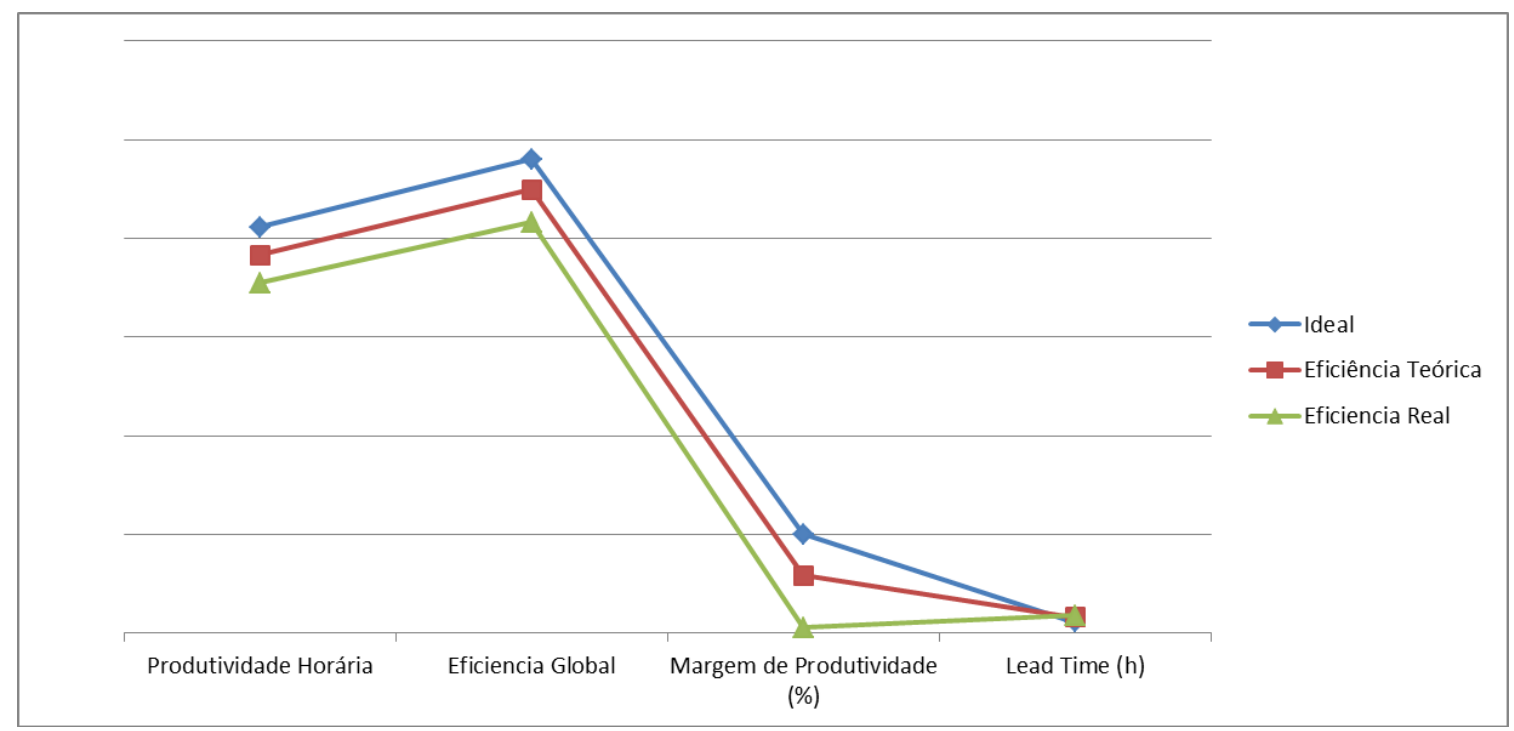

Gráfico 1: Cenários e atributos simulados da linha de usinagem bloco e cabeçote motor

Vale destacar que a eficiência real sempre será menor que a ideal e a técnica. No que se refere ao cenário ideal essa observação é intuitiva devido as estatísticas de falhas não estarem representadas no modelo. Por outro lado a comparação entre o cenário de eficiência técnica e o de eficiência real requer uma análise mais detalhada, pois neste último está contida a contribuição da gestão de manutenção da planta que é imperceptível aos fabricantes de máquinas e equipamentos.

A produtividade horária obtida na simulação atendeu às premissas de volume do escopo do projeto, porém nota-se uma diferença na ordem de aproximadamente $10 \%$ entre os resultados dos dois últimos cenários simulados.

Ao se comparar os cenários com os KPIs produtividade horária, eficiência global e margem de produtividade, é verificada uma relação direta e proporcional entre estes. Porém no que se refere ao KPI Lead Time, esta relação passa a ser inversamente proporcional e não linear, devido ao impacto do comportamento aleatório dos eventos neste. 


\section{CONCLUSÃO}

No setor automotivo percebemos nos últimos anos uma mudança do mercado consumidor que está atento cada vez mais às novidades tecnológicas, lançando assim sobre a indústria automotiva novos desafios para manufaturar tais demandas.

Para o desenvolvimento de um novo processo produtivo a manufatura digital demonstra ser uma ferramental adequada para a geração de conhecimentos que visam o planejamento eficiente e otimizado da manufatura, atendendo assim as demandas do mercado no menor espaço de tempo e custo competitivo.

A disponibilidade de dados de eficiência técnica de máquinas e equipamentos em operação combinados com um simulador resultou em tomadas de decisões relevantes, como por exemplo a inclusão de máquinas (ou a redução), alteração no balanceamento dos ciclos de usinagem, minimização do tempo de bloqueio da operação gargalo e experimentação de diferentes soluções e lógicas de automação para atendimento ao escopo do projeto.

Como contribuição para futuros trabalhos ficou evidente que a exclusiva utilização dos dados de eficiência técnica dos fornecedores não representam as criticidades do processo produtivo, onde a latência da margem de produtividade percentual apresentada pode afetar de forma negativa a eficiência global do sistema de manufatura..

O planejamento da automação de uma linha de usinagem através da simulação a eventos discretos mostrou-se uma alternativa promissora para o conhecimento do comportamento do processo de manufatura sob a ótica de produtividade por conhecer as margens segurança operacionais.

\section{REFERÊNCIAS}

[1] Mourtzis, D.; Doukas, M.; Bernidaki, D. Simulation in Manufacturing: Review and Challenges. Procedia CIRP, Volume 25, Pages 213-229,2014.

[2] ÁlVARES, Alberto José Estudo Dirigido: Métodos para Projeto, Planejamento do Processo e Fabricação de Peças Assistidos por Computador. Disponível em http://www.graco.unb.br/alvares/estudo_dirigido/Planejamento_de_Processo/novo_capp.html \#foot1513. Acesso em: 23 de maio de 2015.

[3] Chang, T.C., Wysk R.A. e Wang, H.P. Computer Aided Manufacturing, Prentice Hall International Series in Industrial and Systems Engineering, W.J. Fabrycky e J.H. Mize (eds.), 2ns Edition, 1998.

[4] LOVE, Jonathan. Process Automation Handbook: A Guide to Theory and Practice. London: Springer Science \& Business Media, 2007.

[5] STAMATIS, D. H. The OEE Primer: Understanding Overall Equipment Effectiveness, Reliability, and Maintainability. London: CRC Press, 2010.

[6] SELEIMA, A.; AZABA, A.; ALGEDDAWYA, T. Simulation Methods for Changeable Manufacturing. 
http://www.sciencedirect.com/science/journal/22128271/3/supp/C. Acesso em: 21 de maio de 2015. 